\title{
GIRALDUS CAMBRENSIS UND DER TOD ARTHURS
}

Während des 12.Jahrhunderts gab es außer William of Newburgh, der die Historia Regum Britanniae des Geoffrey of Monmouth (HRB) in Bausch und Bogen als Fiktion bezeichnete, nur einen weiteren Kritiker, der die Wahrhaftigkeit des Autors und die Verläßlichkeit der mitgeteilten Fakten in Frage stellte: Giraldus Cambrensis. Im Itinerarium Kambriae ${ }^{1}$ berichtet uns der kritische Waliser über einen gewissen Meilerius, der in der Nähe von Urbs Legionum, dem Caerleon der Artussage, wohnte. Dieser Meilerius war von Teufeln besessen, die ihm die Fähigkeit verliehen, zwischen wahr und falsch $\mathrm{zu}$ unterscheiden ${ }^{2}$. Legte man ihm das Evangelium des hl. Johannes auf den Schoß, so verschwanden

1 Giraldi Cambrensis Opera, ed. J.S. Brewer (vols. 1-4), J.F.Dimock (vols. 5-7) and G.F.Warner (vol. 8), Rolls Series, 21 (London, 1861-1891); VI: Itinerarium Kambrix, et Descriptio Kambriæ (1868). Vgl. F.M. Powicke, Gerald of Wales (Manchester, 1928), repr. from The Bulletin of the John Rylands Library, XII, ii (Juli, 1928); Robert H.Fletcher, The Arthurian Material in the Chronicles, 2nd ed. by R.S.Loomis (New York, 1966), S. 180-181. Literatur über Giraldus in: J.Conway Davies, "Giraldus Cambrensis 1146-1946", Archaeologia Cambrensis, The Journal of the Cambrian Archaeological Association, XCIX (1947), 85-108 und 246-280. Wilhelm Kleineke, Englische Fürstenspiegel vom Policraticus Johanns von Salisbury bis zum Basilikon Doron König Jakobs I. (Halle, 1937). G. Phillips und G. Görres, Historisch-politische Blätter für das katholische Deutschland, darin: "Heinrich II. König von England und Giraldus Cambrensis" (München, 1849), S. 689-712. R.H. Fletcher. The Arthurian Material in the Chronicles, darin Bibliographie für die Jahre 1905-1965. Zur Ausgrabung Arthurs vgl. S. 330-331.

2 Vgl. A.G.Prys-Jones, Gerald of Wales - His "Itinerary" through Wales and His "Description" of the Country and Its People (London, 1955), Kap. VI: "Meilyr, the Soothsayer of Caerleon", S. 34-36. 
die Teufel fluchtartig, tauschte man es aber gegen die Historia Regum Britanniae aus, kehrten sie in noch größerer Zahl zurück. Mehrfach spricht Giraldus über die Arturi fabula ${ }^{3}$ und gibt damit zu erkennen, daß er zumindest einige der über Arthur erzählten Geschichten als fiktiv betrachtet. Keineswegs hält Giraldus deshalb aber die gesamte Artusgeschichte bei Geoffrey für erdichtet; er akzeptiert und glaubt vielmehr eine ganze Anzahl von Fakten über Arthur, die er entweder aus der Historia unmittelbar oder aus der reichhaltigen Artustradition, die nicht allein auf Geoffrey zurückgeht, kennenlernte. Wegen seiner kritischen Distanz und der ständigen Neigung zu rationaler Analyse ist Giraldus ein wertvoller Zeuge für das Verhältnis seines Jahrhunderts zu Arthur und zur Artusliteratur, und zwar weniger durch Beibringung neuer, bisher unbekannter Fakten und Daten als durch seine scharfsinnigen und wohlüberlegten Stellungnahmen und Kommentare.

Giraldus sah die Darstellung des Lebens von König Arthur in der $H R B$ in erster Linie als exemplarisch gültigen Modellfall und weniger als historische Quelle an. Gerade die Fürsten sollten sich seiner Ansicht nach intensiv mit den Geschichtsschreibern und ihren Exempla beschäftigen, denn

Historiarum ... lectio vetustarum litterato non mediocriter principi confert, ubi eventus belli varios et aleam incertam, casus asperos et secundos, insidias occultas et cautelas advertere poterit, et de præteritis olim actibus quid aggrediendum, quid vitandum ... tamquam ex speculo, scriptura docente, contemplari ${ }^{4}$.

Die Lektüre alter Historien gereicht einem gebildeten Fürsten zu großem Nutzen. Dort wird er die verschiedenen Kriegsausgänge und den unzuverlässigen Würfel des Schicksals, Unglücks- und Glücksfälle, geheime Intrigen und entsprechende Vorsichtsmaßnahmen erfahren können, und es wird ihm möglich sein, aus längst vergangenen Taten $\mathrm{zu}$ erkennen, was zu tun und was zu lassen ist... so wie aus einem Spiegel, wobei die geschichtliche Quelle die Lehrmeisterin ist.

Giraldus war an Literatur dieser Art also auch aus politischen Gründen interessiert. Entsprechend dem Artusteil der $H R B$ konzipierte und strukturierte er seinen Fürstenspiegel

3 Opera III, 78, 328; IV, 149.

4 Opera VIII, 42-43. 
De Principis Instructione, der im Hauptteil den Casus, die tragedie Heinrichs II. darstellt. Dieses Werk deutet daher die Morte Arthure-Linie voraus. Die Verbindung zwischen Heinrich und Arthur wurde nicht allein von Giraldus geknüpft. Verschiedene Autoren betonen vielmehr, daß die Regierung Heinrichs an die Zeit Arthurs erinnert habe: “...velut ad scolam prudentiae et justitiae, undique confluxerunt; ita ut viderentur Arthuri tempora renovari" ${ }^{5}$.

Auch Heinrich dürfte die Entfaltung und Entwicklung der Artusliteratur aufmerksam verfolgt haben, und zwar nicht nur veranlaßt durch seine Gemahlin Eleanor, die als literarische Mäzenin ständig einen Kreis von Literaten um sich hatte. Heinrich II. selbst war Patron und Förderer von Literatur und Kunst ${ }^{6}$. Marie de France widmete ihm ihre Lais; Joseph von Exeter schrieb einen(nicht erhaltenen) Panegyricus auf den König; Peter von Blois nannte ihn den gebildetsten Mann Europas, Osbert von Clara feierte ihn als Maecenas. Viele führende Historiographen und Dichter kamen mit dem König in persönlichen Kontakt. Einige, wie Walter von Châtillon, Walter Map oder Johann von Tilbury, hielten sich längere Zeit an seinem Hof auf. Gut zwei Drittel der französischen Autoren der Zeit standen mit dem englischen Königshof in Verbindung.

Königin Eleanor wird den ihr gewidmeten Brut des agn. Dichters Wace mit demselben Interesse gelesen haben wie die lyrischen Huldigungen der provençalischen Minnesänger. Heinrich dagegen war mehr an den politischen Implikationen des Artusstoffes interessiert. Bei seinen Überlegungen mußte er zwei Aspekte des Problems im Auge behalten: Hinsichtlich der normannischen und englischen Bevölkerung des Landes, für die Arthur nur einer der frühen Herrscher der Insel war, konnte er einfach in die Fußstapfen des Briten treten; den rebellierenden und immer noch auf die Wiederkehr Arthurs hoffenden Kelten, insbesondere den Walisern, mußte er den

5 Matthaeus Parisiensis, Historia Anglorum, sive ... Historia Minor, ed. F. Madden; Rolls Series, 44, 1-3 (London, 1866-69), I, 397-398.

6 Vgl. W.F.Schirmer, Studien zum literarischen Patronat im England des 12.Jahrhunderts (Köln, 1960), S. 17-22; W.F.Schirmer, Die frühen Darstellungen des Artusstoffes (Köln, 1957), S. 41. 
Glauben an Arthurs Wiederkehr austreiben, wenn er hoffen wollte, in Wales jemals als Souverän anerkannt zu werden. Daß Bretonen, Cornen und Waliser fest an die Wiederkehr des Königs glaubten, wird schon zu Anfang des 12.Jahrhunderts mehrfach bezeugt. In einer Fortsetzung von Gaimars Lestorie des Engles (etwa 1150) ${ }^{7}$ wird uns berichtet, daß die Waliser den Normannen mit Arthur drohten. Wenn der Herrscher das Land wiedererobert hätte, so kündigten sie an, würden alle Normannen verjagt werden, so daß der alte Name Britannien wieder zu seinem Recht käme. Solange das Volk diesen Wunschtraum hegte, mußte es die Verhältnisse im Reich als unangenehmen Übergangszustand betrachten, während dessen es den Nacken gegen die Londoner Usurpatoren steif zu machen galt.

Heinrich beobachtete die Vorgänge in Wales mit äußerster Wachsamkeit ${ }^{8}$. Seine Politik richtete sich darauf, Wales führerlos zu machen. Daher sabotierte er die Ernennung des weltgewandten und diplomatisch geschulten Giraldus zum Bischof von St. David ${ }^{9}$, daher entfachte er Familienfehden, wenn ein Fürst zu mächtig geworden war und im Begriff stand, eine Gefahr für die Sicherheit des Landes zu werden, daher schickte er die besten Köpfe der Waliser ins Ausland oder holte sie an den Londoner Hof. Aber die Wurzel des Übels war damit nicht beseitigt. Heinrich wird sich überlegt haben, wie er den Glauben an den rex futurus zerstören konnte, der dem Widerstandswillen der Waliser eine mythische Legitimation gab.

7 Lestorie des Engles, solum la translacion Maistre Geffrei Gaimar, ed. Th.D.Hardy and Ch.T.Martin, Rolls Series, 91, 1-2 (London, 1888-89), I, 287, V. 244-251:

\footnotetext{
Apertement le vont disant, Forment nus uunt manacant, Ka la parfin tute lauerunt, Par Arthur la receuuerunt $\mathrm{E}$ cest pais tut ensement Toldrunt a la Romeine gent, A la terre sun nun rendreunt; $\mathrm{E}$ Bretaigne lapelerunt.
}

${ }^{8}$ Giraldus Cambrensis, Opera I., 43, 60; III, 49, 53, 109, 156, 168, 229.

๑ Opera I, 42; III, 156. 
Welche Rolle Glastonbury im Artusmythos gespielt hat, kann hier nicht im einzelnen dargelegt werden. Für das Verständnis der Ausgrabungsberichte ist vor allem von Bedeutung, daß die keltische Anderwelt Avallon mit Glastonbury identifiziert wurde und zwar zum ersten Mal von Giraldus Cambrensis:

Quae nunc autem Glastonia dicitur, antiquitus insula Avallonia dicebatur. Est enim quasi insula tota paludibus obsita, unde dicta est Britannice Inis (MS emin) Avallon, id est, insula pomifera. Pomis enim, quae aval Britannica lingua dicuntur, locus ille quondam abundabat. Unde et Morganis, nobilis matrona et partium illarum dominatrix atque patrona, necnon et Arthuro regi sanguine propinqua, post bellum de Kemelen Arthurum ad sanandum ejusdem vulnera in insulam quae nunc Glastonia dicitur deportavit. Dicta quoque quondam Britannice Inis (MS eius) gutrin fuerat, hoc est, insula vitrea; ex quo vocabulo, supervenientes postea Saxones locum illum Glastingeburi vocitabant. Glas enim lingua eorum vitrum sonat, et buri castrum, civitas appellatur ${ }^{10}$.

Was aber jetzt Glastonia heißt, wurde in alten Zeiten Insel Avallon genannt. Denn dieser Ort ist wie eine Insel ganz von Sümpfen umgeben, weshalb er auf britisch Inis Avallon genannt wird; d.h. äpfeltragende Insel. An Äpfeln nämlich, die auf britisch aval heißen, hatte jener Ort einst UUberfluß. Daher brachte auch Morganis, eine edle Frau und Herrin und Beschützerin jener Gegend und sicherlich auch eine Blutsverwandte König Arthurs, nach der Schlacht von Kemelen Arthur zur Heilung seiner Wunden auf diese Insel, die jetzt Glastonia heißt. Auf britisch wurde sie einst auch Inis gutrin genannt, d.h. gläserne Insel. Nach diesem Wort nannten die später dorthin kommenden Sachsen jenen Ort Glastingeburi. Glas heißt nämlich in ihrer Sprache vitrum, und buri castrum, civitas.

Auf britisch wurde Glastonia also nach Giraldus Inis Gutrin, Glasinsel genannt, und nach dieser Bezeichnung sollen die Sachsen den Ort Glastingaburgh genannt haben. Eine ganz ähnliche Erklärung des Ortsnamens finden wir bei William of Malmesbury und Caradoc of Llancarfan. Beide halten Glastonbury für eine Lehnübersetzung des britischen Ynis gutrin, Insula vitrea ${ }^{11}$. Glastonbury lag ursprünglich auf einer

10 De Principis Instructione, Opera VIII, 128. Ähnlich etymologisiert Giraldus in Speculum Ecclesiae (Opera IV, 48-49).

1 Glasinseln sind in der keltischen Literatur schon früh nachzuweisen, z.B. in den "Spoils of Annwyn" [Nr. XXX des Book of Taliesin]. Offenbar handelt es sich dabei um eine Art Paradiesinsel, wie sie 
künstlichen Insel, die von den Kelten zum Schutz vor feindlichen Angriffen in dem großen Seengelände unterhalb des Thor angelegt worden war, eine Art keltisches Venedig ${ }^{12}$. Der Ynis-Bestandteil der keltischen Bezeichnung muß daher durch prähistorische und historische Verhältnisse in und um Glastonbury erklärt werden, wo sich schon viele Jahrhunderte vor der Einwanderung der Sachsen eine blühende keltische Siedlung befand. Offenbar war der Inselcharakter von Glastonbury während des Mittelalters nicht mehr zu erkennen ${ }^{13}$. Auch Giraldus bezweifelt, ob man Glastonbury als insula bezeichnen dürfe:

mediamnis magis proprie diceretur, quoniam mediis amnibus sita est, sicut melius insulae dicuntur, quae salo, hoc est in mari, sitae noscuntur ${ }^{14}$.

Sie (die Insel Avallon) sollte richtiger mediamnis - zwischen den Flüssen liegend - genannt werden, da sie inmitten von Flüssen liegt, so wie man insulae - Inseln - besser die nennt, von denen man weiß, daß sie salo, d.h. im Meer liegen.

Jedenfalls waren beide Namen lange Zeit nebeneinander gebräuchlich. Sie wurden im 12. Jahrhundert durch einen dritten, Avallon, ergänzt. Giraldus Cambrensis berichtet, daß die "fabulosi Britones" an Arthurs Fortleben auf Avallon glaubten:

in Chrétien de Troyes' Erec (V. 1945-51) als Herrschaftsbereich des Königs Maheloas beschrieben wird. Vorgedeutet ist diese Stelle in Caradocs Vita Gildae, die Melwas zum König der aestiva regio und von Glastonbury macht, das als sächsisches Äquivalent von Ynis Gutrin aufgefaßt wird. Dabei kann die umstrittene Frage der etymologischen Ableitung des Namens Glastingaburh außer acht gelassen werden. Vgl. E.Ekwalls Deutungsversuch in The Concise Oxford Dictionary of English Place-Names, 4th ed. (London, 1960), S. 198.

1: Zur Geschichte von Glastonbury, insbesondere des lake-village, vgl. A. Bulleid and H.St. George Gray, The Glastonbury Lake Village, 2 vols. (ohne Ort, 1911, 1917), insbesondere S. 1-10. Vgl. auch Geoffrey Ashe, King Arthur's Avallon, 2nd ed. (London, 1966).

${ }^{13}$ In frühaltenglischer Zeit stehen die Namen Glastingaburh und Glastingaēg (-ieg) nebeneinander. Vgl. dazu Kenneth Cameron, English Place-Names (London, 1963), S. 113, und Eilert Ekwall, a.a. O., S. 198.

${ }^{14}$ Opera IV, 49 (interpoliert).

Vgl. Monast.-Anglic., tom. 1, S. 2, zit. bei Du Cange im Artikel zu mediamnis. 
Propter hoc fabulosi Britones et eorum cantores fingere solebant, quod dea quaedam fantastica, scilicet et Morganis dicta, corpus Arthuri in insulam detulit Avalloniam ad ejus vulnera sanandunı...15 Deswegen pflegten die Sagen liebenden Briten und ihre Barden die Fiktion vorzutragen, daß eine phantastische Göttin - sie wird auch Morganis genannt - den Körper Arthurs zur Heilung seiner Wunden auf die Insel Avallon gebracht hat...

Dem widerspricht, daß Heinrich von einem in der Geschichte beschlagenen britischen Barden, "ab historico cantore Britone" 16 , Kenntnis vom Grabe Arthurs erhalten haben soll. Die zeitgenössischen keltischen Quellen sprechen nur vom Glauben an die Wiederkehr Arthurs, dessen Tod abgeleugnet wird:

Bet y march. bet $\mathrm{y}$ guythur.

bet $\mathrm{y}$ gugaun cletyfrut, anoeth bid bet $\mathrm{y}$ arthur.

Rhys übersetzt:

A grave for March, a grave for Gwythur/

A grave for Gagawn of the ruddy sword/

Not wise (the thought) a grave for Arthur ${ }^{17}$.

Die Übersetzung dieser Stelle bereitet Schwierigkeiten; es liegen verschiedene differierende Versuche vor. Die wesentliche Aussage aber läuft darauf hinaus, daß es kein Grab für Arthur gibt bzw. daß dessen Grab unbekannt ist. Nach Avallon wurde Arthur zum Ausheilen seiner Wunden gebracht, nicht zur Beerdigung. Die Darstellung des Giraldus von Arthurs Fortleben auf Avallon entspricht eher der volksläufigen Auffassung der keltischen Bevölkerung als der angebliche Bericht des Barden über Arthurs Grab.

Avallon jedenfalls identifiziert Giraldus als erster mit Glastonbury. Nach Avallon war König Arthur zum Ausheilen

15 Speculum Ecclesiae, Opera IV, 49.

16 Opera VIII, 128. Zur Bedeutung von "historico cantore" vgl. R.S. Loomis in Arthurian Material in the Chronicles, S. 331: "they mean: from a Breton singer versed in (or dealing with) history!" Zum walisischen Sänger und Barden vgl. A.G.Prys-Jones, Gerald of Wales, s. 114 .

17 Sir Thomas Malory, Le Morte d'Arthure, introd. by Sir J.Rhys, 2 vols. (London, 1906; repr. 1967), I, xii. Rhys zitiert die Facsimile Edition des Black Book (Oxford, 1888), fol. 34a. 
seiner Wunden gebracht worden, in Glastonbury also mußte man - wenn überhaupt irgendwo - nach seinen sterblichen Überresten suchen.

Das Kloster dürfte auf Heinrichs Ansinnen bereitwillig eingegangen sein, und zwar aus verschiedenen Gründen. Zunächst einmal mußte die Auffindung Arthurs das Prestige Glastonburys heben, das in England lange Zeit als "Roma secunda" galt. Ferner gibt es aus den 70er Jahren verschiedene Dokumente über Geldgeschenke Heinrichs an das Kloster sowie über Belehnungen ${ }^{18}$. Nach dem großen Brand von Glastonbury z.B. erließ Heinrich eine Proklamation, in der er den Wiederaufbau des Klosters versprach ${ }^{19}$. Ob diese Gaben mit den Ausgrabungen in Verbindung stehen, konnte ich nicht verifizieren. Fest steht aber, daß das Kloster gegenüber Heinrich Verpflichtungen besaß.

Das genaue Datum der Ausgrabungen ist unsicher. In der Sekundärliteratur findet man immer wieder das zu späte Datum 1191, das, soweit ich sehe, auf Ralph of Coggeshall (Cronicon, S. 36), Matthaeus Parisiensis (II. 27) oder auf Roger Wendover (I. 203) zurückgeht. Robert of Gloucester bringt die Ausgrabung mit dem großen Brand von 1184 in Verbindung. Capgrave nennt als Termin das Jahr 1170, Higden das Jahr 1177 und John of Fordun das Jahr 1180. Leland nennt, einem Anonymus folgend, das Jahr 1189. Bei den mittelalterlichen Historiographen gibt es also keinen Consensus hinsichtlich des Termins der Ausgrabungen. Bei der Festlegung des Datums ist zu berücksichtigen:

1. daß Abt Henry (de Sully) die Ausgrabungsergebnisse (Giraldus und anderen) vorlegte; Henry war Abt von 1189-93.

2. daß Heinrich II. die Ausgrabungen veranlaßte und die Translatio der Reliquien befahl. Heinrich starb 1189. Ein Datum Mitte bzw. Ende der 80er Jahre, nach dem großen Brand von 1184, bei dem fast die ganze Klosteranlage zerstört wurde, erscheint möglich, wenn nicht gar wahrscheinlich.

$18 \mathrm{Vgl}$. Adam of Domerham, Historia de Rebus Gestis Glastoniensibus, ed. Th. Hearne (Oxford, 1727), S. 303-331.

19 Ibid., S. 336-340. 
Giraldus sagt ausdrücklich, daß König Heinrich II. detaillierte Anweisungen für die Ausgrabungen gab und auch die Stelle bezeichnete, wo die Mönche die sterblichen Überreste Arthurs finden würden. Heinrich wußte genau, bis zu welcher Tiefe man graben mußte, und er kannte auch die Beschaffenheit des Sarges, in dem Arthur bestattet worden war:

... maxime tamen et evidentissime rex Angliae Henricus secundus ... totum monachis indicavit, quod profunde, scilicet in terra per $x$ vi. pedes ad minus corpus invenirent, et non [in] lapideo tumulo sed in quercu cavata ${ }^{20}$.

... am besten und deutlichsten hat dennoch der König von England, Heinrich II., den Mönchen das Ganze angekündigt, daß sie nämlich tief unten - wenigstens $16 \mathrm{Fu} \beta$ in der Erde - einen Körper finden würden, und nicht in einem Steingrab, sondern in einer ausgehöhlten Eiche.

Heinrich hat darüber hinaus auch Anweisungen für die Translatio Arthurs gegeben:

Dictus autem abbas corpore reperto, monitis quoque dicti regis Henrici marmoreum ei sepulchrum fieri fecit egregium, tanquam patrono loci illius praecipuo, qui scilicet ecclesiam illam prae caeteris regni cunctis plus dilexerat, terrisque largis et amplis locupletaverat ${ }^{21}$.

Besagter Abt aber ließ, nachdem der Körper (Leichnam) gefunden worden war - auch aufgrund der Ermahnungen des genannten Königs Heinrich - ihm (i.e. Arthur) ein einzigartiges Marmorgrabmal errichten, so wie dem vortrefflichen Patron jenes Ortes, der nämlich jene Kirche mehr als alle anderen seines Königreiches geliebt und ihr durch reiche und große Ländereien zu Wohlstand verholfen hatte.

Aufgrund der Darstellung Giraldus' müssen wir uns fragen, ob der Name des Abtes oder der des Königs stimmt. Die bisherigen Kritiker haben es für selbstverständlich gehalten, daß Giraldus entweder in dem einen oder anderen Fall irrt. Auch der Name des Abtes Henry de Blois (Abt in Glastonbury von 1126-1171.) wird gelegentlich mit den Ausgrabungen in

20 De Principis Instructione, Opera VIII, 127-128.

21 Speculum Ecclesiae, Opera IV, 51.

22 Vgl. Richard Warner, An History of the Abbey of Glaston and the Town of Glastonbury (Bath, 1826), Appendix (gesondert paginiert), S. L: "Henry II ... acquainted Henry de Blois, at that time abbat 
Verbindung gebracht ${ }^{22}$. Es ist aber nicht einzusehen, warum Heinrich II. nicht schon mit dem designierten Abt von Glastonbury, der sein Blutsverwandter und familiaris war, über Klosterangelegenheiten hätte sprechen sollen. Jedenfalls war es Abt Henry de Sully, der 1192 mit Giraldus Cambrensis sprach und ihn über die Ausgrabungsergebnisse informierte ${ }^{23}$.

Weitere Informationen über die Grabstätte Arthurs erhielten die Mönche in nächtlichen Visionen und Wahrträumen. In der Chronica Maior des Matthäus Paris und auch bei Giraldus Cambrensis heißt es, ein Mönch habe den Wunsch ausgesprochen, an einer bestimmten Stelle des Klosterfriedhofs begraben zu werden. Getreu dem letzten Willen des Mitbruders grub man das Grab und stieß auf die Bestattungsstätte Arthurs ${ }^{24}$. Damit wird nichts anderes behauptet, als daß man Arthurs Grab aus Zufall entdeckt hätte. Unverständlich bliebe dann aber, warum man ein normales Grab fast $6 \mathrm{~m}$ tief hätte graben sollen, denn in dieser Tiefe fanden sich die sterblichen Überreste Arthurs. Der Chronist weiß auch, warum man den König so tief in den Boden senkte: aus Angst vor den Sachsen, denen man zutraute, daß sie sich selbst an dem toten Herrscher noch vergreifen würden.

In 16 Fuß Tiefe stieß man auf einen völlig erhaltenen Eichensarg, der mit Axt und Brecheisen geöffnet werden mußte. Darin fand man die überdimensionalen Knochen eines Menschen. Der Schädel wies eine tiefe Einkerbung auf, wahrscheinlich die Todeswunde. Zwei Drittel des Sarges wurden von den Gebeinen dieser heroischen Gestalt eingenommen, der Rest enthielt kleinere, offenbar weibliche Knochen. Ein Mönch griff nach einer goldenen Haarlocke; sie zerfiel unter

(sic) of Glastonbury, with what he had learn't from the ballad of the bard, and desired him to dig and search after the bones of that great king. Henry de Blois search't as he was ordered ... and found King Arthur's bones towards the close of King Henry's reign'. Warner bemerkt jedoch, daß die Auffindung Arthurs nach Meinung Lelands und anderer erst zur Zeit Richards I. erfolgte.

${ }_{23}$ Vgl. H.E. Butler, The Autobiography of Giraldus Cambrensis (London, 1937), S. 118-119.

${ }^{24}$ So auch bei Roger of Wendover, Chronica, sive Flores historiarum, ed. Henricus O. Coxe, English Historical Doc. (London, 1841), III, 48. 
seiner Hand zu Staub ${ }^{25}$. Zu spät stellte er fest, daß er Guineveres Haar berührt hatte.

Daß Giraldus all diese Dinge selbst gesehen bzw. aus erster Hand erfahren hat, braucht nicht bezweifelt zu werden. Wenn dennoch immer wieder literarische Reminiszenzen auftauchen, liegt das z.T. an der Arbeitsweise Giraldus', der seine Werke in reichem Maße mit dem Ornament des Zitats und der klassischen Anspielung schmückt. Sein Bericht über die Exhumierung Arthurs weist Anklänge an die Darstellung der Ausgrabung Kaiser Karls auf, die durch Kaiser Otto III. (983-1002) um das Jahr 1000 erfolgte. In einer interpolierten Fassung der Geschichten Ademars von Chabannes ${ }^{26}$ (12.Jh.) wird folgendes berichtet:

Quidam vero canonicorum eiusdem loci Adalbertus, cum enormi et procero corpore esset, coronam Caroli quasi pro mensura capiti suo circumponens, inventus est strictiori vertice, coronam amplitudine sua vincentem circulum capitis. Crus proprium etiam ad cruris mensuram regis dimetiens, inventus est brevior, et ipsum eius crus protinus divina virtute confractum est. Qui supervivens annis $\mathrm{XL}$, semper debilis permansit.

Einen der Geistlichen desselben Ortes, Adalbert, traf man zufällig a.n, als er, da er sehr hochgewachsen und schlank war, die Krone Karls wie zum Messen auf sein Haupt setzte, wobei dieses aber zu klein war, und die Krone mit ihrer Größe den Umfang seines Kopfes übertraf. Als er auch sein eigenes Schienbein im Vergleich mit dem des Königs maß, fand man, daß seines kürzer war. Und gerade dieses sein Schienbein wurde auf der Stelle durch göttliche Einwirkung gebrochen. Er lebte zwar noch $40 \mathrm{Jahre}$, blieb aber immer Invalide.

Bei Giraldus Cambrensis heißt es in De Principis Instructione (VIII, 129):

Os enim tibiae ipsius appositum [tibiae] longissimi viri loci, quem et nobis abbas ostendit, et juxta pedem illius terrae affixum, large tribus digitis trans genu ipsius se porrexit. Os etian capitis tanquam ad prodigium vel ostentum capax erat et grossum, adeo ut intercilium et inter oculos spatium palmalem amplitudinem large contineret. Apparebant autem in hoc vulnera decem aut plura... etc. ${ }^{27}$.

25 Er war dabei so übereifrig, daß er in das Grab fiel, "in imum fossae profundae se praecipitem dedit”, Spec. Eccl. IV, 47.

${ }_{26}$ Adhémar de Chabannes (ca. 988-1034); Ademari Historiarum Libri Tres, Migne, PL 141 (Paris, 1853), S. 47-50.

27 Opera VIII, 129. 
Als man das Schienbein desselben (i.e. Arthur) an das Schienbein des größten Mannes des Ortes hielt, den auch uns der Abt zeigte, und es neben dem Fuß jenes Mannes auf die Erde stellte, ragte es leicht um 3 Finger über das Knie desselben hinaus. Auch der Schädelknochen war so wundersam und ungeheuer groß und geräumig, daß der Raum zwischen den Augenbrauen (intercilium) und der zwischen den Augen bei weitem die Größe einer Handfläche hatte. Auf diesem Schädel waren aber zehn oder mehr Wunden zu sehen... etc.

Die beiden Berichte stimmen auf merkwürdige Weise überein; abgemessen werden Kopf und Schienbein, und in beiden Fällen sind die Knochen des abgeschiedenen Helden größer. Ich möchte annehmen, daß der grundgelehrte Waliser auf französische Quellen zurückgeht. So findet sich z.B. ein Bericht über die riesenhafte Körpergröße Karls in der Historia Karoli Magni, die angeblich ein Zeitgenosse, der Erzbischof Turpinus von Reims, tatsächlich aber ein Geistlicher zwischen 1147 und 1168 verfaßt und einem Aachener Dekan gewidmet hat ${ }^{28}$.

Giraldus sieht in der Größe von Arthurs Knochen eine Erfüllung der Prophezeiung Vergils:

Sciendum etiam quod ossa reperta corporis Arthuri tam grandia fuerunt, ut et illud poetae completum in his videri posset:

"Grandiaque effosis mirabitur ossa sepulchris" ${ }^{29}$.

Man sollte auch wissen, daß die gefundenen Gebeine Arthurs so groß gewesen sind, daß auch jenes Dichterwort in ihnen möglicherweise erfüllt worden ist:

"Und in aufgeworfenen Gräbern wird man staunend mächtige Gebeine sehen"'.

Dieser Satz wird nicht nur im Text erörtert, sondern steht auch dem Bericht wie eine Art Motto voran; er könnte für Giraldus Leitbild und Modell eigener Vorstellungen hinsichtlich der Ausgrabung gewesen sein.

Unter dem gewaltigen Grabstein fand man, mit der Schrift nach unten liegend, ein bleiernes Kreuz. Darauf stand zu lesen: "hic iacet sepvltvs inclitvs rex artvrivs in insvla avalonia" 30.

${ }^{28}$ Historia Karoli Magni et Rotholandi, ou Chronique du pseudo-Turpin, ed. Cyril Meredith-Jones (Paris, 1936), S. 175-179.

29 Opera VIII, 128.

30 Text nach dem oftmals gezeichneten angeblich gefundenen Kreuz, 
Der Eindruck, den der Fund machte, muß ungeheuer gewesen sein. Endlich besaß man den Beweis, daß Arthur wirklich gelebt hatte - und daß er wirklich gestorben war. Selbst die größten Gelehrten freuten sich über die Entdeckung; sie alle glaubten an die Echtheit des Kreuzes, das übrigens bis zum 18. Jahrhundert erhalten blieb und oftmals abgebildet wurde. Während des 18.Jahrhunderts verschwand es spurlos und ist seitdem nicht wieder aufgetaucht.

Es dürfte heute wohl nur wenige Archäologen oder Historiker geben, die das Kreuz für echt halten ${ }^{31}$. Allgemein ist man davon überzeugt, daß es sich um eine Fälschung handelt, und zwar um eine sehr geschickte. Zunächst einmal ist die Ortsangabe 'hier auf der Insel Avallon' auf einem Grabkreuz überflüssig. Sie wird eingefügt worden sein, um die Identität von Glastonbury und Avallon augenfällig zu machen. Auch die Bezeichnung rex für Arthurius ist bedenklich. Der historische Arthur - wenn es ihn gegeben hat - war Heerführer, dux bellorum. König wird er erst bei Geoffrey of Monmouth.

Erstaunlich ist die Tatsache, daß man Arthur in einem Eichensarg fand. Keltische Häuptlinge und andere bedeutende Männer waren tatsächlich in roh behauenen Eichenstämmen bestattet worden. Das aber sei damals, so sagen uns die Archäologen, nicht (mehr?) bekannt gewesen. Wir können dieses Problem beiseite lassen, da eine wesentliche Prämisse allgemein anerkannt wird: daß die Ausgrabung von irgendeiner Seite manipuliert und das Kreuz gefälscht worden ist. Wem wurde mit dieser Fälschung gedient?

abgebildet bei Treharne, The Glastonbury Legends: Joseph of Arimathia, The Holy Grail and King Arthur (London, 1967), S. 95.

31 Vgl. jedoch C.A. Ralegh Radford, "Glastonbury Abbey", in The Quest of Arthur's Britain, ed. Geoffrey Ashe (London, 1971), S. $97 \mathrm{ff}$. Radford stellt fest, daß die Form der Buchstaben auf dem Kreuz nicht der Schreibweise des 12.Jahrhunderts entspreche. "These forms are proper to the eleventh century or earlier, rather than to the twelfth", S. 100. Natürlich hat der Fälscher sich bemüht, die Schrift einer vergangenen Epoche archaisierend nachzuahmen. Die Argumentationsweise dieses [und anderer Beiträge der Sammlung] wird eher den "romantic patriot" als den Wissenschaftler ansprechen. 
Offenbar zunächst dem Kloster, das nun mit Besuchern aus aller Welt rechnen konnte, beherbergte es doch in seinen Mauern Grab und Reliquien des berühmten Sagenhelden. Immerhin gab es in Glastonbury schon während des 5. Jahrhunderts ein Kloster mit einer berühmten Kirche, der Church of our Lady, die bis zum großen Brand im Jahre 1184 erhalten blieb. In der Royal Charter Heinrichs II., die anläßlich des Wiederaufbaus erlassen wurde, heißt es sogar, Glastonbury sei 'Mutter und auch Grabstätte der Heiligen', 'erbaut von den Jüngern des Herrn' ${ }^{32}$. In Glastonbury hat man sich für Legendenbildung und -tradierung immer sehr aufgeschlossen gezeigt. Noch heute wird z.B. von einem bekannten Geistlichen ein in vielen Auflagen erschienenes Buch vertrieben, in welchem die These vertreten wird, daß Christus selbst Glastonbury besuchte - allerdings: "Our Lord's stay at this place would have been strictly private and pass unnoticed" 33 . Ähnlich wird das Kloster damals die Gelegenheit begrüßt haben, seinen Namen mit dem berühmten Arthur zu verbinden.

Aber auch König Heinrich II. brachte die Fälschung Nutzen, denn er hatte nun den regen futurum endlich im sicheren Grabe. Damit war, so durfte Heinrich erwarten, jener Mythos zerstört, der bei den Walisern trotz militärischer Niederlagen den Glauben an einen mit Arthur erkämpfbaren Sieg wachgehalten hatte.

Heinrich kam vor allem der Umstand zugute, daß das Kloster von 1178 bis 1189 ohne Abt war und während dieser Zeit direkt der Krone (und das heißt: ihm selbst) unterstand. Thomas Hearne schreibt darüber:

Robert Pricr of Winchester ... died anno 1178. After his Death there was no Abbat elected all the reign of Henry the second, but this Abbey was in the King's Hand under custody first of Peter de Marcy, a Monk of Cluny, who died anno 1184, in which year the whole Monastery, except Part of the Abbat's Lodgings and the Steeple, was consumed by Fire. After which the King sent one of

32 Adam von Domerham, Historia de Rebus Gestis Glastoniensibus, S. 337.

${ }^{33}$ Rev. C.C.Dobson, Did Our Lord Visit Britain as They Say in Cornwall and Somerset, 7th ed. (Glastonbury, 1959), S. 42. 
his Chamberlains, Ralph Fitz Stephen, to take care of the Revenues of the Abbey, who began, and in great part finished, a new Church and the Offices of the House, which were perfected by 39 . Henry de Saliaco, or de Soilli, ... who was made Abbat in the Year 1189, being the first Year of the Reign of King Richard the first. In his Time the Tomb of the famous King Arthur was found in the Cemetery, and by the Care of this Abbat (tho' others, with less Probability, say 'twas done in the time of his Predecessor Henry de Blois) it was translated into the Abbey Church, and a noble Monument was erected to his Memory, on which were certain Verses fixed, whereof this Abbat is reported to have been the Author ${ }^{34}$.

Eine gute Zusammenarbeit mit dem Kloster war also dadurch gewährleistet, daß Heinrichs cambellanus und Intimus dort die Geschäfte führte ${ }^{35}$. Radulphus Filius Stephani wird in den Pipe Rolls häufig in Verbindung mit der camera regis genannt, so daß es unwahrscheinlich ist, daß das Kloster die Ausgrabung der Gebeine Arthurs Mitte bis Ende der achtziger Jahre aus eigener Initiative durchgeführt hat. Fand die Ausgrabung aber 1191 statt (wie mehrere Historiker annehmen), so muß eine Teilnahme Heinrichs an der Planung ebenfalls als wahrscheinlich angenommen werden. Der neue Abt Heinrich von Sully war, wie gesagt, Blutsverwandter und Vertrauter des Königs ${ }^{36}$. Es ist daher möglich, daß der König die Aktion mit dem prospektiven Abt geplant und vorbereitet hat. Aber wer es auch war, der die Ausgrabung veranlaßte oder leitete: er hatte offenbar guten Grund, den Ausgrabungsort mit dichten cortinis zu verhängen, um allzu Wissensdurstige an der Beobachtung der Vorgänge zu hindern ${ }^{37}$.

${ }^{34}$ Thomas Hearne, The History and Antiquities of Glastonbury (Oxford, 1722).

35 Vgl. Pipe Rolls, Vol. XXXVIII AD. 1187-88 (London, 1925) passim. Vgl. besonders S. 158: Radulphus filius Stephani redd. comp. de $x l l$. de scutagio [ = Ersatz für ritterlichen Dienst] militum de Glastingeberia quos recognoscit se debere regi. In thesauro liberavit. Et quietus est.

${ }^{36} \mathrm{Vgl}$. J.Leland, Assertio Inclytissimi Arturii Regis Britanniae (Londoni, 1544), ed. Th. Hearne (Londoni, 1770), V, 50: “...monachi anonymi verba huc adducam: 'Conditus hic fuit rex Arturius, sicut per regem Henricum secundum abbas Henricus didicerat, cuius consanguineus \& dudum familiaris exstiterat".

37 Ibid., S. 51: "Anno Domini 1189. quadam die locum cortinis circundans (sic) fodere praecepit." 
Bemerkenswert scheint mir auch der Grabspruch, den Abt Heinrich von Sully nach dem Zeugnis mehrerer Autoren für das Marmorgrab geschrieben haben soll:

On the West Side of the Tomb, that is to say, where King Arthur's Bones were deposited, there was engraven this Inscription:

Hic jacet Arturus, flos Regum, gloria Regni, Quem mores, probitas commendant laude perenni.

And on the East Side, were Queen Guinever's were placed, there was this Inscription:

Arturi jacet hic conjux tumulata secunda

Quæ meruit colos virtutum prole secunda ${ }^{38}$.

Auch Abt Heinrich bezeichnet Guinevere also als zweite Frau Arthurs. Dadurch erhalten die Angaben des Giraldus, die man vielleicht als Irrtum abtun könnte, eine fast offizielle Bestätigung; seine Transkription weicht nämlich hinsichtlich der zweiten Frau von dem Text ab, der auf dem bis ins 18. Jahrhundert erhaltenen, angeblich bei den Ausgrabungen gefundenen Kreuz steht.

Giraldus betont, daß er das Kreuz selbst gesehen und dio Buchstaben selbst gelesen habe: "Crucem autem hanc extractam a lapide, dicto abbate Henrico ostendente, prospeximus, et literas has legimus" 39. Im Speculum Ecclesiae zitiert Giraldus die Inschrift folgendermaßen: "Hic jacet sepultus inclytus rex Arthurius, in insula Avallonia, cum Wennevereia uxore sua secunda" ${ }^{40}$. In De Principis Instructione lautet sie: "Hic jacet sepultus inclitus rex Arthurus cum Wenneuereia vxore sua secunda in insula Avallonia" "41.

Beide Transkriptionen enthalten den Zusatz "cum Wennevereia uxore sua secunda", der auf dem Bleikreuz fehlt. Im Speculum-Zitat wurde die Ergänzung angehängt, in De Principis Instructione eingeschoben. Das letztere Werk erläutert unmittelbar im Anschluß an die Grabschrift die Angabe über die zweite Frau Arthurs:

38 Hearne, The History, S. 157-158. Adam of Domerham zitiert denselben Grabspruch; allerdings heißt die letzte Zeile bei ihm: "Quæ meruit coelos virtutum prole fecunda" (statt "secunda"). Vgl. Historia de Rebus Gestis Glastoniensibus, ed. Th.Hearne, 2 vols. (Oxford, 1727), II, 342.

s9 Opera IV, 50.

40 Ebd.

41 Opera VIII, 127. 
Occurrunt hic autem notabilia plurima; habuerat enim uxores duas, quarum ultima simul cum ipso sepulta fuerat, et ossa ipsius cum ossibus viri simul inventa...42

Hier aber begegnet sehr viel Bemerkenswertes. Er hatte nämlich zwei Frauen, deren letzte mit ihm zusammen bestattet worden ist und deren Gebeine zugleich mit denen ihres Mannes gefunden wurden.

Ein Irrtum ist also nicht möglich: Giraldus hat ein anderes Kreuz gesehen als das später ausgestellte.

Die Überlieferungsgeschichte der Kreuzesinschrift ist außerordentlich verwirrend. Die verschiedenen Texte widersprechen einander; hielte man sie für historisch verbürgt, hätte es nicht nur zwei, sondern eine Vielzahl von Kreuzen geben müssen.

So gibt etwa Henry Knighton eine mit der Giraldus-Inschrift nahezu identische an: "Hic jacet sepultus inclitus rex Arthurus cum Genevera uxore sua secunda in insula Avalona" 43. Eine leicht verkürzte Fassung findet sich im Eulogium, einer Chronik des 14.Jahrhunderts: "Arthurus rex cum uxore sua regina jacent hic sepulti" 44. Die von Roger von Wendover und Matthäus Paris zitierte Aufschrift kommt der des noch Jahrhunderte später ausgestellten Kreuzes am nächsten; bei ihnen heißt es: "Hic jacet inclytus Britonum rex Arthurus, in insula Avalonis sepultus" ${ }^{45}$. Ein Kuriosum unter den Inschriften stellt die zweite im Eulogium enthaltene Version dar: "Hic jacet inclytus Arthurus a Mordredo proditore occisus" 46 . Es lassen sich also deutlich drei Traditionen er-

42 Ebd.

43 Chronicon, ed. J.R. Lumby, Rolls Series, 92, 1-2 (London, 1889-95), I, 150. - Bei Ranulf Higden heißt es ebenso; nur der Name der zweiten Frau ist als "Wenevera" wiedergegeben (Polychronicon, ed. J.R.Lumby, Rolls Series, 41, 1-9 [London, 1864-86], VIII, 62).

44 Eulogium (Historiarum sive Temporis): Chronica ab orbe condito usque ad annum Domini M.CCC.LXVI., ed. F.S.Haydon, Rolls Series, 9, 1-3 (London, 1858-63), III, 91.

45 Roger de Wendover, The Flowers of History, ed. Henry G. Hewlett, Rolls Series, 84, 1-3 (London 1886-89), I, 203. Matthäus Paris, Chronica Majora, ed. H.R.Luard, Rolls Series, 57, 1-7 (London, 1872-83), II, 379. - Der Text der Annales de Morgan stimmt fast wörtlich damit überein; vgl. Annales Monastici, ed. H.R.Luard, Rolls Series, 36, 1-5 (London, 1864-69), I, 21. 
kennen: man übernahm entweder die Giraldus-Fassung oder verkürzte sie um den expliziten Hinweis auf Arthurs zweite Frau, oder unterließ überhaupt jeden Hinweis auf eine Frau. Daß dieser Überlieferung keine historische Entwicklung zur kürzeren Fassung unterliegt, belegen spätmittelalterliche Versionen wie z.B. die John Capgraves, der den Giraldus-Text übernahm: "Here lith the nobil Kyng Arthure, with his secunde wyf, Veneraca, in the ylde cleped Avallone" "47.

Die Wiedergabe der Inschrift ist stets eingebettet in einen mehr oder weniger umfangreichen Bericht der Ausgrabungsgeschichte, die in ganz bestimmte Überlieferungsreihen eingeteilt werden kann. Die mittelalterlichen Autoren wollten das ihrer Meinung nach Wesentliche niederschreiben, wobei sie das Recht für sich in Anspruch nahmen, einen bestimmten Passus zu erweitern oder zu kürzen. So findet man etwa in späteren Ausgrabungsberichten zahlreiche Zusätze, die sich auf keine der beiden Giraldus-Versionen stützen können ${ }^{48}$. Da das erhaltene Kreuz eine von Giraldus abweichende Inschrift trug, könnte man vermuten, es handele sich dabei um eine spätere Ausfertigung; allerdings gibt auch Ralph of Coggeshall eine ganz ähnliche Inschrift wieder ${ }^{49}$. Ob das ursprüngliche Kreuz verloren gegangen war oder ob seine Aufschrift - aus welchen Gründen auch immer - verändert wurde, muß offen bleiben. Auf jeden Fall kann man nicht, wie öfter geschehen, aus der späteren Version schließen, Giraldus habe die Inschrift falsch wiedergegeben.

Welche Motive hätte Giraldus haben sollen, den Zusatz: "cum Wennevereia uxore sua secunda" eigenmächtig einzu-

${ }^{46}$ Eulogium, II, 363.

47 John Capgrave (1393-1464), The Chronicle of England, ed. F.Ch. Hingeston; Rolls Series, 1 (London, 1858), S. 140, 141.

48 So ist z.B. in den Annales de Morgan von drei Grabhügeln die Rede: "Primum tumumulum (sicl) dicunt fuisse Guenhavere reginae, uxoris ejusdem Arthuri: secundum Modredi nepotis ejusdem: tertium praedicti principis" (Annales Monastici, I, 22). Offensichtlich sind hier die beiden Grabhügel, die sich nach des Giraldus Bericht über dem Grab Arthurs befanden, mißdeutet worden.

49 Radulphi de Coggeshall. Chronicon Anglicanum, ed. Josephus Stevenson, Rolls Series, 66 (London, 1875), S. 36: "Hic jacet inclitus rex Arturius, in insula Avallonis sepultus". 
fügen? Weder bei Geoffrey of Monmouth noch in den späteren französischen Vers- und Prosaromanen finden wir Guinevere als zweite Frau Arthurs. Wohl hat Arthur in der einen oder anderen Fassung ein illegitimes Kind, aber nirgends eine zweite Frau. Auch daß sich hier die später so beliebte Motivdoppelung (zwei Isolden, zwei Guineveres) vordeutet, ist unwahrscheinlich. Eher könnte darin eine alte keltische Tradition ihren Niederschlag gefunden haben. Nach J.Rhys wird in den walisischen Triaden von Arthur gesagt, er habe nacheinander drei Frauen gehabt, die alle Gwenhwyvar hießen ${ }^{50}$. Nach einer anderen Triade war die 'zweite Gwenhwyfach' an der Schlacht von Camlan - und damit am Untergang der arthurischen Welt - schuld ${ }^{51}$. Es ist durchaus denkbar, daß Giraldus mit einer solchen Geschichte über Guinevere vertraut war. Da er aber nicht an der Echtheit des Fundes zweifelte und sich für die einzelnen Fakten verbürgt, hat ein solcher Erklärungsversuch wenig Überzeugungskraft. Mir scheint die Annahme einer bewußten Fälschung mit politischer Intention wahrscheinlicher. Nur dürfte sie kaum auf das Konto der Mönche gehen, die an einer solchen Veränderung des historischen Materials nicht interessiert gewesen sein können, und erst recht kann sie nicht Giraldus, dem Erzfeind Heinrichs, zugeschrieben werden. Es drängt sich der Verdacht auf, daß Heinrich den Artusmythos für seine Zwecke mißbrauchte.

so J.Rhys, Vorwort zur Malory-Ausgabe, S. xxiv. Vgl. dazu jedoch Rachel Bromwich, Trioedd Ynys Prydein (Cardiff, 1961), S. 154, Triade 56:

The three great Queens of Arthur's court:

Gwennhwyfar daughter of (Cywryd) Gent, and Gwenhwyfar daughter of (Gwythyr) son of Greidiawl and Gwenhwyfar daughter of (G)ogfran the Giant.

R. Bromwich fragt sich, ob die drei Guineveres lediglich "witticism" sind oder ob es sie in der walisischen Tradition tatsächlich gegeben hat. Sie findet Argumente für beide Thesen, neigt allerdings eher der letzteren zu.

51 Triade 53b: "Three harmful Blows of the Island of Britain". Es heißt unter 2: "The second Gwenhwyfach struck upon Gwenhwyfar: and for that cause there took place afterwards the Action of the battle of Camlan." Es wäre möglich, daß es sich bei Gwenhwyfach nur um einen Schreibfehler für Gwenhwyfar handelt. 
Heinrichs 'erste Frau' war Eleanor von Poitou 52. Er scheint sie nicht sonderlich geliebt $\mathrm{zu}$ haben. Verschiedene Geschichtsschreiber deuten an, daß er sie in erster Linie zur Ausweitung seiner Macht und seines französischen Besitzes geehelicht habe. Vor 1174 lebte er bereits in offenem Ehebruch mit Fair Rosamond ${ }^{53}$, einem bedeutungslosen, aber sehr hübschen jungen Mädchen, und versuchte hartnäckig, Eleanor zum Eintritt in ein Kloster zu bewegen, dasselbe Kloster übrigens, in dem er nach seinem Tode bestattet wurde. Schon zu dieser Zeit scheint sich Heinrich mit dem Gedanken an eine neue Ehe getragen zu haben. Seine Pläne nahmen aber erst nach dem überraschenden Tod von Rosamond Gestalt an. Vom französischen Königshof wurde ihm nämlich zu dieser Zeit Alice of France, die Schwester Philips und zukünftige Gemahlin Richards zugeführt, damit sie sich an das fremde Land und den Hof in London gewöhne. Der leidenschaftliche und unbeherrschte König verführte die unerfahrene Braut des Sohnes, und die Affaire drang bald an das Licht der Öffentlichkeit ${ }^{54}$. Philip teilte das Vorgefallene seinem künftigen Schwager mit, der seinen Vater hinfort mit tödlichem $\mathrm{Ha}$ verfolgte.

Heinrich selbst bewahrte, wie in allen Familienangelegenheiten, unerschütterliche Ruhe. Er setzte sich mit Rom in Verbindung und forderte den Besuch des päpstlichen Legaten Uguccione Pier Leoni an, mit dem er über die Scheidung von Eleanor und die Hochzeit mit Richards Braut beraten wollte. Zugunsten der aus dieser Ehe ihm geschenkten Kinder wollte er die mit Eleanor gezeugten enterben ${ }^{55}$. Der Zeitpunkt für diese Ereignisse liegt etwa in der Mitte der 80er Jahre, könnte also mit den Vorbereitungen für die Ausgrabung zusammenfallen. Auf diese Weise wäre zu erklären, warum auf dem Grabkreuz die sonst nirgends bekannte zweite Gemahlin

52 Zum Verhältnis Heinrichs zu Eleanor vgl. Jaques Boussard, Le Gouvernement d'Henri II Plantagenêt (Paris, 1956), S. 483, 487, 514, $544,571$.

53 Vgl. De Principis Instructione, Opera VIII, 165.

54 Ebd., S. 232. Vgl. dazu jedoch: Richard Barber, Henry Plantagenet. A Biography (London, 1964), S. 227.

${ }_{55}$ De Principis Instructione, Opera VIII, 232. 
Arthurs erwähnt wird. Die zu erwartende zweite Ehe Heinrichs sollte durch die Parallele im Artusmythos legitimiert, werden. Die Kriege in Frankreich nahmen jedoch bald die ganze Aufmerksamkeit Heinrichs in Anspruch; daher kam es nicht zur Ausführung des in allen Einzelheiten wohl vorbereiteten Plans.

Die Ausgrabung der Gebeine Arthurs hatte erstaunliche Auswirkungen. Keineswegs räumte sie mit dem Wiederkehrglauben endgültig auf. Die Legende von Arthurs Fortleben auf Avallon existierte in der mündlichen Tradition und in der Literatur weiter, so z.B. im Werk des berühmt-berüchtigten Huchown of the Awle Ryale, dessen Gest Historialle nicht erhalten ist. Wyntoun, der das Werk noch gelesen hat, schrieb um 1400 in seiner eigenen Chronik ${ }^{56}$ :

Bot off hys dede and hys last end

I fand na wryt, couth mak that kend (V. 4367-8).

Daher will er nur das sagen, was er bei Huchown fand (V.4370):

Bot quhen that he bade fowychtyn fast, Efftyre in till an Ile he past

Sare woundyt, to be lechyd thare,

And efftyre he wes se[yn] na mare" (V. 4371-74).

Bei Hector Boece heißt es (noch 1531-35):

In no storie autentik that I reid,

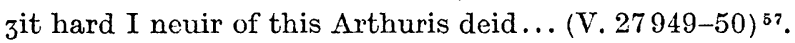

Wie die Daten dieser Texte zeigen, hatte selbst die zweite Ausgrabung der Gebeine Arthurs den Mythos von seiner Wiederkehr nicht zerstören können. Adam von Domerham, der spätere Chronist des Klosters Glastonbury, war Augenzeuge dieser zweiten Ausgrabung, die am 19. April 1277 in Anwesenheit von König Edward I. und dessen Gemahlin Eleanor erfolgte. Edward, der ähnlich wie Heinrich mit den Walisern viel Ärger hatte, ließ ein prächtiges Grabmal für Arthur errichten. Adam of Domerham las bei dieser Gelegen-

36 Androw of Wyntoun, The Orygynale Cronykyl of Scotland, ed. D. Laing, 3 vols. (Edinburgh, 1872-79), V. $4345 \mathrm{ff}$.

57 Hector Boece, The Buik of the Croniclis of Scotland ..., ed. W.B. Turnbull, Rolls Series, 6, 1-3 (London, 1858), II, 261. 
heit den Heinrich von Sully zugeschriebenen Grabspruch ${ }^{58}$. Albericus Trium Fontium (Albéric des Trois Fontaines), der die erste kontinentale Version der Ausgrabung Arthurs schrieb, berichtet 1236 über die Auffindung eines steinernen Grabes mit einem Deckel aus Blei ("lamina plumbea"), auf dem die Worte standen:

Hic jacet Arturus flos regum, gloria regni

Quem probitas morum commendat laude perenni.

Hic jacet Arturus Britonum rex ultor inultus etc. (sic) ${ }^{59}$

Hier liegt Arthur, die Zierde der Könige, der Ruhm des Reiches,

Den die Lauterkeit seines Charakters mit ewigem Lobe rühmt.

Hier liegt Arthur, der Briten König, der ungerächte Rächer.

Jedenfalls hat sich bei den Historiographen, wie zu erwarten, die Morte Arthure-Version durchgesetzt, was für die Artus-Literatur der Folgezeit bedeutsam wurde. Immer bestimmter wird die Kritik am Wiederkehrglauben, den zahlreiche Autoren als lächerlichen Aberglauben der Briten mit Hohn und Spott abtun. Schon Joseph von Exeter gibt in seinem De Bello Trojano den Tenor dieser später vorherrschenden Richtung an:

Sic Britonum ridenda fides, et credulus error

Arturum expectat expectabitque perenne ... ${ }^{60}$

So erwartet der Briten lächerlicher Glaube und argloser Irrtum

Arthur und wird ihn immer erwarten...

Wenn jemand gegen die Vernunft oder die Natur etwas Unwahrscheinliches erhoffte, wurde er mit den auf Arthurs Rückkehr wartenden Briten verglichen:

5s Historia de Rebus Gestis Glastoniensibus, ed. Th.Hearne, 2 vols. (Oxford, 1727), II, 342. Vgl. John Leland, The Itinerary, 5 vols. (London, 1964), I, 288. Bei Hearne heißt es: "Henry de Saliaco, or de Soilli ... H. de Juliaco, and in others Henry Swansey". Hearne, The History and Antiquities, S. 91-92.

59 "Chronica Albrici Monachi Trium Fontium", ed. Paulus SchefferBoichorst, MG, ss. XXIII, S. 871.

${ }^{60}$ Dictys Cretensis et Dares Phrygius de Bello Trojano etc. Josephi Iscani De Bello Trojano Libri Sex a Samuele Dresemio Dithmarso (Londini, 1825), S. 464, V. 472-73. 
Qui cupit auferre naturam seminat herbam

Cujus in Arturi tempora fructus erit ${ }^{61}$.

Wer die Natur vertreiben will, sät cin Korn, das zu Arthurs Zeiten Frucht tragen wird.

Et prius Arturus veniet vetus ille Britannus Quam ferat adversis falsus amicus opem ${ }^{62}$. Eher wird Arthur kommen, jener alte Brite, Als daß ein falscher Freund im Unglück Hilfe bringt.

John Maior sagt expressis verbis:

Unde in fabulam ductum est, quando aliquis qui numquam veniet, expectatur, hunc expectas sicut Arthurum Britanni ${ }^{63}$.

Von daher ist es zum geflügelten Wort geworden, da $B$ man, wenn man jemanden erwartet, der nie kommen wird, diesen erwartet wie die Briten Arthur.

In der europäischen Literatur entstand nach der "Auffindung" der Gebeine Arthurs ein neuer Seitenzweig der matière de Bretagne, die Morte Arthure-Tradition. Das erste Werk dieser Richtung ist der Perlesvaus eines unbekannten Dichters; der Bericht des Giraldus über die Ausgrabung wird in wesentlichen Punkten in dieses Werk aufgenommen. Weitere Werke nennen sich sogar Morte Arthure, wie z.B. der me. stabende Morte Arthure (ein weiteres lateinisches Werk dieser Richtung wurde kürzlich in einer englischen Bibliothek entdeckt). Arthur stirbt in all diesen Erzählungen eines natürlichen, wenn auch gewaltsamen Todes und wird in Glastonbury beigesetzt. Sein Charakter verändert sich: der ehedem übermenschliche nationale Heros wird zum Menschen. Heinrich hatte scheinbar sein Ziel erreicht.

Es müßte daher diejenigen, die ihr Weltbild auf der Prämisse der kritischen Rationalität des Menschen aufbauen, bedenklich stimmen, wenn sie als Kolophon des berühmten Allit. Morte Arthure lesen:

Hic jacet Arthurus rex quondam rexque futurus ${ }^{64}$.

${ }^{61}$ Polycarpy Leyseri ... Historia Poetarum et poematum Medii Aevi Decem (Halae-Magdeburg, 1721), S. 460.

62 Ebd., S. 477.

${ }^{63}$ Historia Majoris Britanniae, Tam Angliae quam Scotiae, per Joannem Majorem, nomine quidem Scotum, professione autem Theologum, e veterum monumentis concinnata (Edinburgh, 1740), S. 67.

${ }_{64}$ Morte Arthure or the Death of Arthur, ed. Edmund Brock, EETS, OS 8 (London, 1871; repr. 1961), S. 128. 
Und es ist noch gar nicht so lange her, daß eine Gruppe von Historikern Cadbury Castle in der Nähe von Glastonbury besuchte und von einem alten Mann gefragt wurde: "Have you come to take the King out?" 65

REGENSBURG

KarL Heinz GöLleR

65 J.A. Robinson, Two Glastonbury Legends (London, 1921), S. 53. 